\title{
MacMahon-type Identities for Signed Even Permutations
}

\author{
Dan Bernstein \\ Department of Mathematics \\ The Weizmann Institute of Science, Rehovot 76100, Israel \\ dan. bernstein@weizmann.ac.il
}

Submitted: May 21, 2004; Accepted: Nov 15, 2004; Published: Nov 22, 2004

Mathematics Subject Classifications: 05A15, 05A19

\begin{abstract}
MacMahon's classic theorem states that the length and major index statistics are equidistributed on the symmetric group $S_{n}$. By defining natural analogues or generalizations of those statistics, similar equidistribution results have been obtained for the alternating group $A_{n}$ by Regev and Roichman, for the hyperoctahedral group $B_{n}$ by Adin, Brenti and Roichman, and for the group of even-signed permutations $D_{n}$ by Biagioli. We prove analogues of MacMahon's equidistribution theorem for the group of signed even permutations and for its subgroup of even-signed even permutations.
\end{abstract}

\section{Introduction}

A classic theorem by MacMahon [6] states that two permutation statistics, namely the length (or inversion number) and the major index, are equidistributed on the symmetric group $S_{n}$. Many refinements and generalizations of this theorem are known today (see 8] for a brief review). In [8], Regev and Roichman gave an analogue of MacMahon's theorem for the alternating group $A_{n} \subseteq S_{n}$, and in [1], Adin, Brenti and Roichman gave an analogue for the hyperoctahedral group $B_{n}=C_{2} \imath S_{n}$. Both results involve natural generalizations of the $S_{n}$ statistics having the equidistribution property.

Our main result here (Proposition 4.1) is an analogue of MacMahon's equidistribution theorem for the group of signed even permutations $L_{n}=C_{2} \succ A_{n} \subseteq B_{n}$. Namely, we define two statistics on $L_{n}$, the $L$-length and the negative alternating reverse major index, and show that they have the same generating function, hence they are equidistributed. Our Main Lemma (Lemma 4.6) shows that every element of $L_{n}$ has a unique decomposition into a descent-free factor and a signless even factor.

In 3], Biagioli proved an analogue of MacMahon's theorem for the group of even-signed permutations $D_{n}$ (signed permutations with an even number of sign changes). Using 
our main result, we prove an analogue for the group of even-signed even permutations $(L \cap D)_{n}=L_{n} \cap D_{n}$ (see Proposition 5.2).

The rest of this paper is organized as follows: Section 2 contains a review of wreath products and known results concerning generators and canonical presentations in $S_{n}, B_{n}$ and $A_{n}$. In Section 3 we define the group $L_{n}$, introduce a canonical presentation in $L_{n}$, and define the statistics we use. In Section 4 we prove the equidistribution property for $L_{n}$, and in Section 5 we prove the equidistribution property for $(L \cap D)_{n}$. Finally, in Section 6, we note three open problems.

\section{Preliminaries}

\subsection{Notation}

For an integer $a \geq 0$ we let $[a]=\{1,2, \ldots, a\}$ (where $[0]=\emptyset)$.

Let $C_{a}$ be the cyclic group of order $a$.

Let $S_{n}$ be the symmetric group on $1, \ldots, n$ and let $A_{n} \subset S_{n}$ denote the alternating group.

\subsection{Wreath Products}

Let $G$ be a group and let $A$ be a subgroup of $S_{n}$. Recall that the wreath product $G$ < $A$ is the group $\left\{\left(\left(g_{1}, \ldots, g_{n}\right), v\right) \mid g_{i} \in G, v \in A\right\}$ with multiplication given by

$$
\left(\left(g_{1}, \ldots, g_{n}\right), v\right)\left(\left(h_{1}, \ldots, h_{n}\right), w\right)=\left(\left(g_{1} h_{v^{-1}(1)}, \ldots, g_{n} h_{v^{-1}(n)}\right), v w\right) .
$$

The order of $G$ < $A$ is $|G|^{n}|A|$.

Let $X=G \times[n]$. For $\left(\left(g_{1}, \ldots, g_{n}\right), v\right) \in G \curlywedge A$, define $f_{\left(\left(g_{1}, \ldots, g_{n}\right), v\right)}: X \rightarrow X$ by

$$
f_{\left(\left(g_{1}, \ldots, g_{n}\right), v\right)}(h, i):=\left(h g_{v(i)}, v(i)\right) .
$$

One can verify that if $G$ is Abelian, then function composition is compatible with mul-

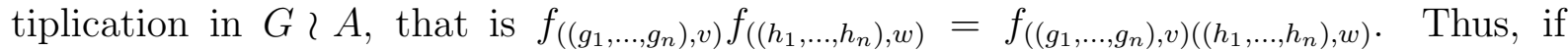
$G$ is Abelian we can identify $\left(\left(g_{1}, \ldots, g_{n}\right), v\right)$ with $f_{\left(\left(g_{1}, \ldots, g_{n}\right), v\right)}$ and we can write $\pi=$ $\left(\left(g_{1}, \ldots, g_{n}\right), v\right) \in G<A$ as

$$
\pi=\left[f_{\pi}(1,1), f_{\pi}(1,2), \ldots, f_{\pi}(1, n)\right]=\left[\left(g_{v(1)}, v(1)\right), \ldots,\left(g_{v(n)}, v(n)\right)\right] .
$$

Call this the window notation of $\pi$.

\subsubsection{The Group of Signed Permutations}

If $G=C_{2}=\{-1,1\}$, then we write $X$ simply as $\{ \pm 1, \pm 2, \ldots, \pm n\}$ and identify every $\sigma \in C_{2}$ 乙 $A$ with a bijection of $X$ onto itself satisfying $\sigma(-i)=-\sigma(i)$ for all $i \in[n]$. We write $\sigma=\left[\sigma_{1}, \ldots, \sigma_{n}\right]$ to mean that $\sigma(i)=\sigma_{i}$ for $i \in[n]$.

In particular, the hyperoctahedral group $B_{n}:=C_{2} 2 S_{n}$ is the group of all bijections of $\{ \pm 1, \pm 2, \ldots, \pm n\}$ satisfying the above condition. It is also known as the group of signed permutations. 


\subsection{Generators and Canonical Presentation}

In this subsection we review generators and canonical presentations in the groups $S_{n}, B_{n}$ and $A_{n+1}$.

\subsection{1 $S_{n}$}

The Coxeter System of $S_{n} . S_{n}$ is a Coxeter group of type $A$. The Coxeter generators are the adjacent transpositions $\left\{s_{i}\right\}_{i=1}^{n-1}$ where $s_{i}:=(i, i+1)$. The defining relations are the Moore-Coxeter relations:

$$
\begin{aligned}
& \left(s_{i} s_{i+1}\right)^{3}=1 \quad(1 \leq i<n), \\
& \left(s_{i} s_{j}\right)^{2}=1 \quad(|i-j|>1), \\
& s_{i}^{2}=1 \quad(1 \leq i<n) .
\end{aligned}
$$

The $S$ Canonical Presentation. The following presentation of elements in $S_{n}$ by Coxeter generators is well known (see for example [5, pp. 61-62]).

For each $1 \leq j \leq n-1$ define

$$
R_{j}^{S}:=\left\{1, s_{j}, s_{j} s_{j-1}, \ldots, s_{j} s_{j-1} \cdots s_{1}\right\},
$$

and note that $R_{1}^{S}, \ldots, R_{n-1}^{S} \subseteq S_{n}$.

Theorem 2.1 (see [5, pp. 61-62]). Let $w \in S_{n}$. Then there exist unique elements $w_{j} \in$ $R_{j}^{S}, 1 \leq j \leq n-1$, such that $w=w_{1} \ldots w_{n-1}$. Thus, the presentation $w=w_{1} \ldots w_{n-1}$ is unique.

For a proof, see for example [8, Section 3.1].

Definition 2.2 (see [8, Definition 3.2]). Call $w=w_{1} \ldots w_{n-1}$ in the above theorem the $S$ canonical presentation of $w \in S_{n}$.

\subsection{2 $B_{n}$}

The Coxeter System of $B_{n} . B_{n}$ is a Coxeter group of type $B$, generated by $s_{1}, \ldots, s_{n-1}$ together with an exceptional generator $s_{0}:=[-1,2,3, \ldots, n]$, whose action is as follows:

$$
\begin{aligned}
{\left[\sigma_{1}, \sigma_{2}, \ldots, \sigma_{n}\right] s_{0} } & =\left[-\sigma_{1}, \sigma_{2}, \ldots, \sigma_{n}\right] \\
s_{0}\left[\sigma_{1}, \ldots, \pm 1, \ldots, \sigma_{n}\right] & =\left[\sigma_{1}, \ldots, \mp 1, \ldots, \sigma_{n}\right]
\end{aligned}
$$

(see [4, §8.1]). The additional relations are: $s_{0}^{2}=1,\left(s_{0} s_{1}\right)^{4}=1$, and $s_{0} s_{i}=s_{i} s_{0}$ for all $1<i<n$.

The $B$ Canonical Presentation. For each $0 \leq j \leq n-1$ define

$$
\begin{array}{r}
R_{j}^{B}:=\left\{1, s_{j}, s_{j} s_{j-1}, \ldots, s_{j} s_{j-1} \cdots s_{1}, s_{j} s_{j-1} \cdots s_{1} s_{0},\right. \\
\left.s_{j} s_{j-1} \cdots s_{1} s_{0} s_{1}, \ldots, s_{j} s_{j-1} \cdots s_{1} s_{0} s_{1} \cdots s_{j}\right\},
\end{array}
$$

and note that $R_{0}^{B}, \ldots, R_{n-1}^{B} \subseteq B_{n}$.

The following theorem is the case $a=2$ of [9, Propositions 3.1 and 3.3]. For a proof of the general case, see for example [2, Ch. 3.3]. 
Theorem 2.3. Let $\sigma \in B_{n}$. Then there exist unique elements $\sigma_{j} \in R_{j}^{B}, 0 \leq j \leq n-1$, such that $\sigma=\sigma_{0} \ldots \sigma_{n-1}$. Moreover, written explicitly $\sigma_{0} \ldots \sigma_{n-1}=s_{i_{1}} s_{i_{2}} \ldots s_{i_{r}}$ is a reduced expression for $\sigma$, that is $r$ is the minimum length of an expression of $\sigma$ as a product of elements in $\left\{s_{i}\right\}_{i=0}^{n-1}$.

Definition 2.4. Call $\sigma=\sigma_{0} \ldots \sigma_{n-1}$ in the above theorem the $B$ canonical presentation of $\sigma \in B_{n}$.

Remark 2.5. For $\sigma \in S_{n}$, the $B$ canonical presentation of $\sigma$ coincides with its $S$ canonical presentation.

Example 2.6. Let $\sigma=[5,-1,2,-3,4]$, then $\sigma_{4}=s_{4} s_{3} s_{2} s_{1} ; \sigma \sigma_{4}^{-1}=[-1,2,-3,4,5]$, therefore $\sigma_{3}=1$ and $\sigma_{2}=s_{2} s_{1} s_{0} s_{1} s_{2}$; and finally $\sigma \sigma_{4}^{-1} \sigma_{3}^{-1} \sigma_{2}^{-1}=[-1,2,3,4,5]$ so $\sigma_{1}=1$ and $\sigma_{0}=s_{0}$. Thus $\sigma=\sigma_{0} \sigma_{1} \sigma_{2} \sigma_{3} \sigma_{4}=\left(s_{0}\right)(1)\left(s_{2} s_{1} s_{0} s_{1} s_{2}\right)(1)\left(s_{4} s_{3} s_{2} s_{1}\right)$.

\subsection{3 $A_{n+1}$}

A Generating Set for $A_{n+1}$. Let

$$
a_{i}:=s_{1} s_{i+1} \quad(1 \leq i \leq n-1) .
$$

The set $A=\left\{a_{i}\right\}_{i=1}^{n-1}$ generates $A_{n+1}$. This set has appeared in [7], where it is shown that the generators satisfy the relations

$$
\begin{array}{rlrl}
\left(a_{i} a_{j}\right)^{2}=1 & & (|i-j|>1), \\
\left(a_{i} a_{i+1}\right)^{3}=1 & & (1 \leq i<n-1), \\
a_{i}^{2}=1 & & (1<i \leq n-1), \\
a_{1}^{3}=1 &
\end{array}
$$

(see [7, Proposition 2.5]).

Note that $\left(A_{n+1}, A\right)$ is not a Coxeter system (in fact, $A_{n+1}$ is not a Coxeter group) as $a_{1}^{2} \neq 1$.

The $A$ Canonical Presentation. The following presentation of elements in $A_{n+1}$ by generators from $A$ has appeared in [8, Section 3.3].

For each $1 \leq j \leq n-1$ define

$$
R_{j}^{A}:=\left\{1, a_{j}, a_{j} a_{j-1}, \ldots, a_{j} \cdots a_{2}, a_{j} \cdots a_{2} a_{1}, a_{j} \cdots a_{2} a_{1}^{-1}\right\},
$$

and note that $R_{1}^{A}, \ldots, R_{n-1}^{A} \subseteq A_{n+1}$.

Theorem 2.7 (see [8, Theorem 3.4]). Let $v \in A_{n+1}$. Then there exist unique elements $v_{j} \in R_{j}^{A}, 1 \leq j \leq n-1$, such that $v=v_{1} \ldots v_{n-1}$, and this presentation is unique.

Definition 2.8 (see [8, Definition 3.5]). Call $v=v_{1} \ldots v_{n-1}$ in the above theorem the $A$ canonical presentation of $v \in A_{n+1}$. 


\section{The Group of Signed Even Permutations}

Our main object of interest in this paper is the group $L_{n}:=C_{2} \succ A_{n}$. It is the subgroup of $B_{n}$ of index 2 containing the signed even permutations (which is not to be confused with the group of even-signed permutations mentioned in Section (5). The order of $L_{n}$ is $\left|C_{2}\right|^{n}\left|A_{n}\right|=2^{n-1} n !$

Example $3.1\left(L_{3}\right)$. Table 1 lists all the elements of $L_{3}$ (in window notation) with their $B$ and $L$ canonical presentation and $B$ - and $L$-length (defined in the sequel).

\begin{tabular}{|c|c|c|c|c|}
\hline$\pi$ & $B$ canonical presentation & $\ell_{B}(\pi)$ & $L$ canonical presentation & $\ell_{L}(\pi)$ \\
\hline$+1,+2,+3]$ & 1 & 0 & 1 & 0 \\
\hline$-1,+2,+3]$ & $\left(s_{0}\right)$ & 1 & $\left(a_{0}\right)$ & 1 \\
\hline$+1,-2,+3]$ & $\left(s_{1} s_{0} s_{1}\right)$ & 3 & $\left(a_{1} a_{0} a_{1}^{-1}\right)$ & 2 \\
\hline$-1,-2,+3]$ & $\left(s_{0}\right)\left(s_{1} s_{0} s_{1}\right)$ & 4 & $\left(a_{0} a_{1} a_{0} a_{1}^{-1}\right)$ & 3 \\
\hline$+1,+2,-3]$ & $\left(s_{2} s_{1} s_{0} s_{1} s_{2}\right)$ & 5 & $\left(a_{1}^{-1} a_{0} a_{1}\right)$ & 4 \\
\hline$-1,+2,-3]$ & $\left(s_{0}\right)\left(s_{2} s_{1} s_{0} s_{1} s_{2}\right)$ & 6 & $\left(a_{0}\right)\left(a_{1}^{-1} a_{0} a_{1}\right)$ & 5 \\
\hline$+1,-2,-3]$ & $\left(s_{1} s_{0} s_{1}\right)\left(s_{2} s_{1} s_{0} s_{1} s_{2}\right)$ & 8 & $\left(a_{1} a_{0} a_{1}^{-1}\right)\left(a_{1}^{-1} a_{0} a_{1}\right)$ & 6 \\
\hline$-1,-2,-3]$ & $\left(s_{0}\right)\left(s_{1} s_{0} s_{1}\right)\left(s_{2} s_{1} s_{0} s_{1} s_{2}\right)$ & 9 & $\left(a_{0} a_{1} a_{0} a_{1}^{-1}\right)\left(a_{1}^{-1} a_{0} a_{1}\right)$ & 7 \\
\hline$+2,+3,+1]$ & $\left(s_{1}\right)\left(s_{2}\right)$ & 2 & $\left(a_{1}\right)$ & 1 \\
\hline$-2,+3,+1]$ & $\left(s_{1} s_{0}\right)\left(s_{2}\right)$ & 3 & $\left(a_{1} a_{0} a_{1}^{-1}\right)\left(a_{1}\right)$ & 3 \\
\hline$+2,-3,+1]$ & $\left(s_{1}\right)\left(s_{2} s_{1} s_{0} s_{1}\right)$ & 5 & $\left(a_{1}^{-1} a_{0} a_{1}^{-1}\right)$ & 4 \\
\hline$-2,-3,+1]$ & $\left(s_{1} s_{0}\right)\left(s_{2} s_{1} s_{0} s_{1}\right)$ & 6 & $\left(a_{1} a_{0} a_{1}^{-1}\right)\left(a_{1}^{-1} a_{0} a_{1}^{-1}\right)$ & 5 \\
\hline$+2,+3,-1]$ & $\left(s_{0}\right)\left(s_{1}\right)\left(s_{2}\right)$ & 3 & $\left(a_{0}\right)\left(a_{1}\right)$ & 2 \\
\hline$-2,+3,-1]$ & $\left(s_{0}\right)\left(s_{1} s_{0}\right)\left(s_{2}\right)$ & 4 & $\left(a_{0} a_{1} a_{0} a_{1}^{-1}\right)\left(a_{1}\right)$ & 4 \\
\hline$+2,-3,-1]$ & $\left(s_{0}\right)\left(s_{1}\right)\left(s_{2} s_{1} s_{0} s_{1}\right)$ & 6 & $\left(a_{0}\right)\left(a_{1}^{-1} a_{0} a_{1}^{-1}\right)$ & 5 \\
\hline$-2,-3,-1]$ & $\left(s_{0}\right)\left(s_{1} s_{0}\right)\left(s_{2} s_{1} s_{0} s_{1}\right)$ & 7 & $\left(a_{0} a_{1} a_{0} a_{1}^{-1}\right)\left(a_{1}^{-1} a_{0} a_{1}^{-1}\right)$ & 6 \\
\hline$+3,+1,+2]$ & $\left(s_{2} s_{1}\right)$ & 2 & $\left(a_{1}^{-1}\right)$ & 1 \\
\hline$-3,+1,+2]$ & $\left(s_{2} s_{1} s_{0}\right)$ & 3 & $\left(a_{1}^{-1} a_{0}\right)$ & 3 \\
\hline$+3,-1,+2]$ & $\left(s_{0}\right)\left(s_{2} s_{1}\right)$ & 3 & $\left(a_{0}\right)\left(a_{1}^{-1}\right)$ & 2 \\
\hline$-3,-1,+2]$ & $\left(s_{0}\right)\left(s_{2} s_{1} s_{0}\right)$ & 4 & $\left(a_{0}\right)\left(a_{1}^{-1} a_{0}\right)$ & 4 \\
\hline$+3,+1,-2]$ & $\left(s_{1} s_{0} s_{1}\right)\left(s_{2} s_{1}\right)$ & 5 & $\left(a_{1} a_{0} a_{1}^{-1}\right)\left(a_{1}^{-1}\right)$ & 3 \\
\hline$-3,+1,-2]$ & $\left(s_{1} s_{0} s_{1}\right)\left(s_{2} s_{1} s_{0}\right)$ & 6 & $\left(a_{1} a_{0} a_{1}^{-1}\right)\left(a_{1}^{-1} a_{0}\right)$ & 6 \\
\hline$+3,-1,-2]$ & $\left(s_{0}\right)\left(s_{1} s_{0} s_{1}\right)\left(s_{2} s_{1}\right)$ & 6 & $\left(a_{0} a_{1} a_{0} a_{1}^{-1}\right)\left(a_{1}^{-1}\right)$ & 4 \\
\hline$-3,-1,-2]$ & $\left(s_{0}\right)\left(s_{1} s_{0} s_{1}\right)\left(s_{2} s_{1} s_{0}\right)$ & 7 & $\left(a_{0} a_{1} a_{0} a_{1}^{-1}\right)\left(a_{1}^{-1} a_{0}\right)$ & 7 \\
\hline
\end{tabular}

Table 1: $L_{3}$

\subsection{Characterization in Terms of the $B$ Canonical Presentation}

Define the group homomorphism abs: $C_{2} \prec S_{n} \rightarrow S_{n}$ by $\left(\left(\epsilon_{1}, \ldots, \epsilon_{n}\right), \sigma\right) \mapsto \sigma$, or equivalently, in terms of our representation of elements of $C_{2}\left\{S_{n}\right.$ as bijections of $\{ \pm 1, \ldots, \pm n\}$ onto itself, $\operatorname{abs}(\sigma)(i):=|\sigma(i)|$. 
From this formulation one sees immediately that for any $\sigma \in B_{n}$, $\operatorname{abs}\left(\sigma s_{0}\right)=\operatorname{abs}(\sigma)$. Thus if $\sigma=s_{i_{1}} \ldots s_{i_{k}}$, then deleting all occurrences of $s_{0}$ from $s_{i_{1}} \ldots s_{i_{k}}$ what remains is an expression for $\operatorname{abs}(\sigma)$. Since by definition $\operatorname{abs}\left(L_{n}\right)=A_{n}$, we have the following proposition.

Proposition 3.2.

$$
L_{n}=\left\{\sigma \in B_{n} \mid \sigma=s_{i_{1}} \ldots s_{i_{k}}, \#\left\{j \mid i_{j} \neq 0\right\} \text { is even }\right\} .
$$

\subsection{Generators and Canonical Presentation}

\subsubsection{A Generating Set for $L_{n+1}$}

$L_{n+1}$ is generated by $a_{1}, \ldots, a_{n-1}$ together with the generator $a_{0}:=s_{0}=[-1,2,3, \ldots, n, n+$ 1]. The additional relations are $a_{0}^{2}=1,\left(a_{0} a_{1}\right)^{6}=\left(a_{0} a_{1}^{-1}\right)^{6}=1$, and $\left(a_{0} a_{i}\right)^{4}=1$ for all $1<i \leq n-1$.

\subsubsection{The $L$ Canonical Presentation}

Let $R_{0}^{L}:=\left\{1, a_{0}, a_{1} a_{0} a_{1}^{-1}, a_{0} a_{1} a_{0} a_{1}^{-1}\right\}$ and for each $1 \leq j \leq n-1$ define

$$
\begin{aligned}
R_{j}^{L}:= & R_{j}^{A} \cup\left\{a_{j} a_{j-1} \cdots a_{2} a_{1}^{-1} a_{0}, a_{j} a_{j-1} \cdots a_{2} a_{1}^{-1} a_{0} a_{1}^{-1}\right\} \\
& \cup\left\{a_{j} a_{j-1} \cdots a_{2} a_{1}^{-1} a_{0} a_{1}, \ldots, a_{j} a_{j-1} \cdots a_{2} a_{1}^{-1} a_{0} a_{1} a_{2} \cdots a_{j}\right\} .
\end{aligned}
$$

For example,

$$
R_{2}^{L}=\left\{1, a_{2}, a_{2} a_{1}, a_{2} a_{1}^{-1}, a_{2} a_{1}^{-1} a_{0}, a_{2} a_{1}^{-1} a_{0} a_{1}^{-1}, a_{2} a_{1}^{-1} a_{0} a_{1}, a_{2} a_{1}^{-1} a_{0} a_{1} a_{2}\right\} .
$$

Note that $R_{0}^{L}, \ldots, R_{n-1}^{L} \subseteq L_{n+1}$.

Theorem 3.3. Let $\pi \in L_{n+1}$. Then there exist unique elements $\pi_{j} \in R_{j}^{L}, 0 \leq j \leq n-1$, such that $\pi=\pi_{0} \ldots \pi_{n-1}$, and this presentation is unique.

A proof is given below.

Definition 3.4. Call $\pi=\pi_{0} \ldots \pi_{n-1}$ in the above theorem the $L$ canonical presentation of $\pi \in L_{n+1}$.

The following recursive L-Procedure is a way to calculate the $L$ canonical presentation:

First note that $R_{0}^{L}=L_{2}$ so $R_{0}^{L}$ gives the canonical presentations of all $\pi \in L_{2}$.

For $n>1$, let $\pi \in L_{n+1},|\pi(r)|=n+1$.

If $\pi(r)=n+1$, 'pull $n+1$ to its place on the right' by

$$
\begin{aligned}
{[\ldots, n+1, \ldots] a_{r-1} a_{r} \cdots a_{n-1} } & =[\ldots \ldots, n+1] & & \text { if } r>2 \\
{[k, n+1, \ldots] a_{1}^{-1} a_{2} \cdots a_{n-1} } & =[\ldots \ldots, n+1] & & \text { if } r=2 \\
\text { (*) } \quad[n+1, \ldots] a_{1} a_{2} \cdots a_{n-1} & =[\ldots \ldots, n+1] & & \text { if } r=1
\end{aligned}
$$


and if $\pi(r)=-(n+1)$, 'correct the sign' by

$$
\begin{aligned}
{[\ldots,-(n+1), \ldots] a_{r-2} \cdots a_{1}^{-1} a_{0}=[n+1, \ldots] } & \text { if } r>3, \\
{[\ell, k,-(n+1), \ldots] a_{1}^{-1} a_{0}=[n+1, \ldots] } & \text { if } r=3, \\
{[k,-(n+1), \ldots] a_{1} a_{0}=[n+1, \ldots] } & \text { if } r=2 \\
{[-(n+1), \ldots] a_{0}=[n+1, \ldots] } & \text { if } r=1
\end{aligned}
$$

and then 'pull to the right' using $(*)$.

This gives $\pi_{n-1} \in R_{n-1}^{L}$ and $\pi \pi_{n-1}^{-1} \in L_{n}$. Therefore by induction $\pi=\pi_{0} \ldots \pi_{n-2} \pi_{n-1}$ with $\pi_{j} \in R_{j}^{L}$ for all $0 \leq j \leq n-1$.

For example, let $\pi=[3,5,-4,2,-1]$, then $\pi_{3}=a_{3} a_{2} a_{1} ; \pi \pi_{3}^{-1}=[-4,3,2,-1,5]$, therefore $\pi_{2}=a_{2} a_{1}^{-1} a_{0}$; next $\pi \pi_{3}^{-1} \pi_{2}^{-1}=[2,3,-1,4,5]$ so $\pi_{1}=a_{1} ;$ and finally $\pi \pi_{3}^{-1} \pi_{2}^{-1} \pi_{1}^{-1}=$ $[-1,2,3,4,5]$ so $\pi_{0}=a_{0}$. Thus

$$
\pi=\pi_{0} \pi_{1} \pi_{2} \pi_{3}=\left(a_{0}\right)\left(a_{1}\right)\left(a_{2} a_{1}^{-1} a_{0}\right)\left(a_{3} a_{2} a_{1}\right) .
$$

Table 1 gives the $L$ canonical presentation of $L_{3}$.

Proof of Theorem 3.9. The L-Procedure proves the existence of such a presentation, and the uniqueness follows by a counting argument:

$$
\prod_{j=0}^{n-1}\left|R_{j}^{L}\right|=\prod_{j=0}^{n-1} 2(j+2)=2^{n}(n+1) !=2^{n+1}\left|A_{n+1}\right|=\left|L_{n+1}\right| .
$$

Remark 3.5. For $\pi \in A_{n+1}$, the $L$ canonical presentation of $\pi$ coincides with its $A$ canonical presentation.

Remark 3.6. The canonical presentation of $\pi \in L_{n+1}$ is not necessarily a reduced expression. For example, the canonical presentation of $\pi=[-3,1,-2] \in L_{3}$ is $\pi=$ $\left(a_{1} a_{0} a_{1}^{-1}\right)\left(a_{1}^{-1} a_{0}\right)$ which is not reduced $\left(\pi=a_{1} a_{0} a_{1} a_{0}\right)$.

\section{3 $\quad B_{n}$ and $L_{n+1}$ Statistics}

Definition 3.7. Let $w=\left[w_{1}, w_{2}, \ldots, w_{n}\right]$ be a word on $\mathbb{Z}$. The inversion number of $w$ is defined $\operatorname{as} \operatorname{inv}(w):=\#\left\{1 \leq i<j \leq n \mid w_{i}>w_{j}\right\}$.

For example, $\operatorname{inv}([5,-1,2,-3,4])=6$.

Definition 3.8. 1. Let $\sigma \in B_{n}$, then $j \geq 2$ is a l.t.r.min (left-to-right minimum) of $\sigma$ if $\sigma(i)>\sigma(j)$ for all $1 \leq i<j$.

2. Define $\operatorname{del}_{B}(\sigma):=\# \operatorname{ltrm}(\sigma)=\#\{2 \leq j \leq n \mid j$ is a l.t.r.min of $\sigma\}$.

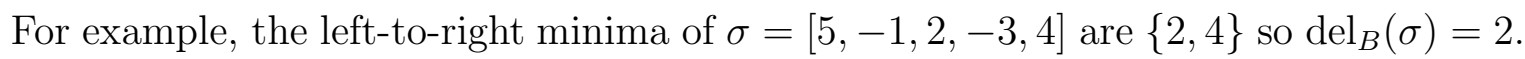

Remark 3.9. The implicit definition of $\operatorname{del}_{S}(w)$ for $w \in S_{n}$ in [8, Proposition 7.2] is similar to the above definition of $\operatorname{del}_{B}$. In particular, if $w \in S_{n}$ then $\operatorname{del}_{S}(w)=\operatorname{del}_{B}(w)$. 
Definition 3.10. Let $\sigma \in B_{n}$. Define

$$
\operatorname{Neg}(\sigma):=\{i \in[n] \mid \sigma(i)<0\} .
$$

Remark 3.11. 1. If $v \in S_{n}$ and $\sigma \in B_{n}$ then

$$
\begin{aligned}
\operatorname{Neg}(v \sigma) & =\{i \in[n] \mid v(\sigma(i))<0\} \\
& =\{i \in[n] \mid \sigma(i)<0\} \\
& =\operatorname{Neg}(\sigma) .
\end{aligned}
$$

2. $\operatorname{Neg}\left(\sigma^{-1}\right)=\{|\sigma(i)| \mid i \in \operatorname{Neg}(\sigma)\}$.

Definition 3.12. Let $\sigma \in B_{n}$. Define the $B$-length of $\sigma$ in the usual way, i.e., $\ell_{B}(\sigma)$ is the length of $\sigma$ with respect to the Coxeter generators of $B_{n}$.

For example,

$$
\ell_{B}([5,-1,2,-3,4])=\ell_{B}\left(s_{0} s_{2} s_{1} s_{0} s_{1} s_{2} s_{4} s_{3} s_{2} s_{1}\right)=10
$$

(see Example 2.6).

Lemma 3.13 (see [4, §8.1]). Let $\sigma \in B_{n}$. Then

$$
\ell_{B}(\sigma)=\operatorname{inv}(\sigma)+\sum_{i \in \operatorname{Neg}\left(\sigma^{-1}\right)} i
$$

In [8], the $A$-length of $w \in A_{n}, \ell_{A}(w)$ was defined as the length of $w$ 's $A$ canonical presentation, and it was shown to have the following property.

Proposition 3.14 (see [8, Proposition 4.4]). Let $w \in A_{n}$, then

$$
\ell_{A}(w)=\ell_{S}(w)-\operatorname{del}_{S}(w),
$$

where $\ell_{S}(w)$ is the length of $w$ with respect to the Coxeter generators of $S_{n}$.

This serves as motivation for the following definition.

Definition 3.15. Let $\sigma \in B_{n}$. Define the $L$-length of $\sigma$ as

$$
\ell_{L}(\sigma):=\ell_{B}(\sigma)-\operatorname{del}_{B}(\sigma)=\operatorname{inv}(\sigma)-\operatorname{del}_{B}(\sigma)+\sum_{i \in \operatorname{Neg}\left(\sigma^{-1}\right)} i .
$$

Remark 3.16. 1. The function $\ell_{L}$ is not a length function with respect to any set of generators, that is for every set of generators of $L_{n}$, there exists $\pi \in L_{n}$ such that $\ell_{L}(\pi)$ is in not the length of a reduced expression for $\pi$ using those generators. For example, in $L_{3}$ we have $\ell_{L}([3,1,2])=\ell_{L}([-1,2,3])=1$ but $\ell_{L}([3,1,2][-1,2,3])=\ell_{L}([-3,1,2])=3$.

2. If $w \in A_{n}$ then, according to Proposition 3.14 and the above remarks, $\ell_{A}(w)=$ $\ell_{L}(w)$. 
Definition 3.17. 1. The $S$-descent set of $\sigma \in B_{n}$ is defined by

$$
\operatorname{Des}_{S}(\sigma):=\{1 \leq i \leq n-1 \mid \sigma(i)>\sigma(i+1)\} .
$$

2. Define the major index of $\sigma \in B_{n}$ by

$$
\operatorname{maj}_{B}(\sigma):=\sum_{i \in \operatorname{Des}_{S}(\sigma)} i
$$

3. Define the reverse major index of $\sigma \in B_{n}$ by

$$
\operatorname{rmaj}_{B_{n}}(\sigma):=\sum_{i \in \operatorname{Des}_{S}(\sigma)}(n-i) .
$$

For example, if $\sigma=[5,-1,2,-3,4]$ then $\operatorname{Des}_{S}(\sigma)=\{1,3\}, \operatorname{maj}_{B}(\sigma)=4$ and $\operatorname{rmaj}_{B_{5}}(\sigma)=$ 6 .

Remark 3.18. $\operatorname{Des}_{S}(\sigma)=\left\{1 \leq i \leq n-1 \mid \ell_{B}\left(\sigma s_{i}\right)<\ell_{B}(\sigma)\right\}$. Indeed, by Remark 3.11 and the definition of inv, for $1 \leq i \leq n-1$

$$
\begin{aligned}
\ell_{B}\left(\sigma s_{i}\right)-\ell_{B}(\sigma) & =\left(\operatorname{inv}\left(\sigma s_{i}\right)+\sum_{i \in \operatorname{Neg}\left(\left(\sigma s_{i}\right)^{-1}\right)} i\right)-\left(\operatorname{inv}(\sigma)+\sum_{i \in \operatorname{Neg}\left(\sigma^{-1}\right)} i\right) \\
& =\operatorname{inv}\left(\sigma s_{i}\right)-\operatorname{inv}(\sigma) \\
& = \begin{cases}+1 & \text { if } \sigma(i)<\sigma(i+1), \\
-1 & \text { if } \sigma(i)>\sigma(i+1) .\end{cases}
\end{aligned}
$$
shows.

The $\mathrm{maj}_{B}$ and $\mathrm{rmaj}_{B_{n}}$ statistics are equidistributed on $B_{n}$, as the following lemma

Lemma 3.19. There exists an involution $\phi$ of $B_{n}$ satisfying the conditions

$$
\operatorname{maj}_{B}(\sigma)=\operatorname{rmaj}_{B_{n}}(\phi(\sigma))
$$

and

$$
\operatorname{Neg}\left(\sigma^{-1}\right)=\operatorname{Neg}\left((\phi(\sigma))^{-1}\right) . .
$$

Proof. Given $\sigma=\left[\sigma_{1}, \ldots, \sigma_{n}\right] \in B_{n}, \sigma_{i_{1}}<\sigma_{i_{2}}<\cdots<\sigma_{i_{n}}$, let $\rho_{\sigma}$ be the order-reversing permutation on $\left\{\sigma_{1}, \ldots, \sigma_{n}\right\}$, that is $\rho_{\sigma}\left(\sigma_{i_{k}}\right)=\sigma_{i_{n+1-k}}$, and define

$$
\phi(\sigma)=\left[\rho_{\sigma}\left(\sigma_{n}\right), \rho_{\sigma}\left(\sigma_{n-1}\right), \ldots, \rho_{\sigma}\left(\sigma_{1}\right)\right] .
$$

Since $\rho_{\sigma}$ is a permutation, the letters in the window notation of $\phi(\sigma)$ are again $\sigma_{1}, \ldots, \sigma_{n}$, so $\rho_{\phi(\sigma)}=\rho_{\sigma}$. Thus

$$
\begin{aligned}
\phi^{2}(\sigma) & =\left[\rho_{\phi(\sigma)}\left(\rho_{\sigma}\left(\sigma_{1}\right)\right), \ldots, \rho_{\phi(\sigma)}\left(\rho_{\sigma}\left(\sigma_{n}\right)\right)\right] \\
& =\left[\rho_{\sigma}^{2}\left(\sigma_{1}\right), \ldots, \rho_{\sigma}^{2}\left(\sigma_{n}\right)\right] \\
& =\sigma
\end{aligned}
$$


and by Remark 3.11, $\operatorname{Neg}\left(\sigma^{-1}\right)=\operatorname{Neg}\left(\phi(\sigma)^{-1}\right)$.

Finally,

$$
\begin{aligned}
i \in \operatorname{Des}_{S}(\phi(\sigma)) & \Longleftrightarrow \phi(\sigma)(i)>\phi(\sigma)(i+1) \\
& \Longleftrightarrow \rho_{\sigma}\left(\sigma_{n+1-i}\right)>\rho_{\sigma}\left(\sigma_{n-i}\right) \\
& \Longleftrightarrow \sigma_{n+1-i}<\sigma_{n-i} \\
& \Longleftrightarrow n-i \in \operatorname{Des}_{S}(\sigma),
\end{aligned}
$$

So

$$
\operatorname{rmaj}_{B_{n}}(\phi(\sigma))=\sum_{i \in \operatorname{Des}_{S}(\phi(\sigma))} n-i=\sum_{i \in \operatorname{Des}_{S}(\sigma)} i=\operatorname{maj}_{B}(\sigma) .
$$

Example 3.20. Let $\sigma=[5,-1,2,-3,4]$. To compute $\phi(\sigma)$, we first reverse $\sigma$ to get $[4,-3,2,-1,5]$, then apply the order-reversing permutation on $\{-3,-1,2,4,5\}$ to get $\phi(\sigma)=[-1,5,2,4,-3]$. Indeed we have $\operatorname{maj}_{B}(\sigma)=4=\operatorname{rmaj}_{B_{5}}(\phi(\sigma))$ and $\operatorname{Neg}\left(\sigma^{-1}\right)=$ $\{1,3\}=\operatorname{Neg}\left(\phi(\sigma)^{-1}\right)$.

Definition 3.21. 1. The A-descent set of $\pi \in L_{n+1}$ is defined by

$$
\operatorname{Des}_{A}(\pi):=\left\{1 \leq i \leq n-1 \mid \ell_{L}\left(\pi a_{i}\right) \leq \ell_{L}(\pi)\right\},
$$

and the $A$-descent number of $\pi \in L_{n+1}$ is defined by $\operatorname{des}_{A}(\pi):=\left|\operatorname{Des}_{A} \pi\right|$.

2. Define the alternating reverse major index of $\pi \in L_{n+1}$ by

$$
\operatorname{rmaj}_{L_{n+1}}(\pi):=\sum_{i \in \operatorname{Des}_{A}(\pi)}(n-i)
$$

3. Define the negative alternating reverse major index of $\pi \in L_{n+1}$ by

$$
\operatorname{nrmaj}_{L_{n+1}}(\pi):=\operatorname{rmaj}_{L_{n+1}}(\pi)+\sum_{i \in \operatorname{Neg}\left(\pi^{-1}\right)} i .
$$

For example, if $\pi=[5,-1,2,-3,4]$ then $\operatorname{Des}_{A}(\pi)=\{1,2\}, \operatorname{rmaj}_{L_{5}}(\pi)=5$, and $\operatorname{nrmaj}_{L_{5}}(\pi)=5+1+3=9$.

Remark 3.22. 1. For $w \in A_{n+1}$, the above definitions agree with [8, Definition 1.5].

2. In general, $\operatorname{Des}_{A}(\pi) \neq\{1 \leq i \leq n-1 \mid \pi(i)>\pi(i+1)\}$.

\section{Equidistribution on $L_{n+1}$}

The following is our main result.

Proposition 4.1. For every $B \subseteq[n+1]$

$$
\begin{aligned}
\sum_{\left\{\pi \in L_{n+1} \mid \operatorname{Neg}\left(\pi^{-1}\right) \subseteq B\right\}} q^{\mathrm{nrmaj}_{L_{n+1}}(\pi)}=\sum_{\left\{\pi \in L_{n+1} \mid \operatorname{Neg}\left(\pi^{-1}\right) \subseteq B\right\}} q^{\ell_{L}(\pi)} \\
=\prod_{i \in B}\left(1+q^{i}\right) \prod_{i=1}^{n-1}\left(1+q+\cdots+q^{i-1}+2 q^{i}\right) .
\end{aligned}
$$


Example 4.2. For $n=3$ and $B=\{2\}$ we have

$$
\begin{gathered}
\sum_{\left\{\pi \in L_{4} \mid \operatorname{Neg}\left(\pi^{-1}\right) \subseteq\{2\}\right\}} q^{\mathrm{nrmaj}_{L_{4}}(\pi)}=\sum_{\left\{\pi \in L_{4} \mid \operatorname{Neg}\left(\pi^{-1}\right) \subseteq\{2\}\right\}} q^{\ell_{L}(\pi)} \\
=\left(1+q^{2}\right)(1+2 q)\left(1+q+2 q^{2}\right)=1+3 q+5 q^{2}+7 q^{3}+4 q^{4}+4 q^{5},
\end{gathered}
$$

as one may verify using Table 2 .

\begin{tabular}{ccc}
$\pi$ & nrmaj $_{L_{4}}(\pi)$ & $\ell_{L}(\pi)$ \\
\hline$[+1,+2,+3,+4]$ & 0 & 0 \\
{$[+1,+3,+4,+2]$} & 1 & 2 \\
{$[+1,+4,+2,+3]$} & 2 & 2 \\
{$[+2,+1,+4,+3]$} & 1 & 1 \\
{$[+2,+3,+1,+4]$} & 2 & 1 \\
{$[+2,+4,+3,+1]$} & 3 & 3 \\
{$[+3,+1,+2,+4]$} & 2 & 1 \\
{$[+3,+2,+4,+1]$} & 1 & 2 \\
{$[+3,+4,+1,+2]$} & 3 & 3 \\
{$[+4,+1,+3,+2]$} & 3 & 3 \\
{$[+4,+2,+1,+3]$} & 2 & 2 \\
{$[+4,+3,+2,+1]$} & 3 & 3 \\
{$[+1,-2,+3,+4]$} & 2 & 2 \\
{$[+1,+3,+4,-2]$} & 3 & 4 \\
{$[+1,+4,-2,+3]$} & 4 & 4 \\
{$[-2,+1,+4,+3]$} & 3 & 3 \\
{$[-2,+3,+1,+4]$} & 4 & 3 \\
{$[-2,+4,+3,+1]$} & 5 & 5 \\
{$[+3,+1,-2,+4]$} & 4 & 3 \\
{$[+3,-2,+4,+1]$} & 3 & 4 \\
{$[+3,+4,+1,-2]$} & 5 & 5 \\
{$[+4,+1,+3,-2]$} & 5 & 5 \\
{$[+4,-2,+1,+3]$} & 4 & 4 \\
{$[+4,+3,-2,+1]$} & 5 & 5
\end{tabular}

Table 2: $\left\{\pi \in L_{4} \mid \operatorname{Neg}\left(\pi^{-1}\right) \subseteq\{2\}\right\}$

By the Inclusion-Exclusion Principle we have:

Corollary 4.3. For every $B \subseteq[n+1]$

$$
\sum_{\left\{\pi \in L_{n+1} \mid \operatorname{Neg}\left(\pi^{-1}\right)=B\right\}} q^{\text {nrmaj }_{L_{n+1}}(\pi)}=\sum_{\left\{\pi \in L_{n+1} \mid \operatorname{Neg}\left(\pi^{-1}\right)=B\right\}} q^{\ell_{L}(\pi)} .
$$


Note that the case $B=\emptyset$ of Proposition 4.1 is just the case $t=1$ of the following theorem.

\section{Theorem 4.4 (see [8, Theorem 6.1(2)]).}

$$
\begin{aligned}
\sum_{w \in A_{n+1}} q^{\ell_{A}(w)} t^{\operatorname{del}_{A}(w)}= & \sum_{w \in A_{n+1}} q^{\operatorname{rmaj}_{A_{n+1}}(w)} t^{\operatorname{del}_{A}(w)} \\
& =(1+2 q t)\left(1+q+2 q^{2} t\right) \cdots\left(1+q+\cdots+q^{n-2}+2 q^{n-1} t\right) .
\end{aligned}
$$

The proof of Proposition 4.1 uses the decomposition of

$$
\left\{\pi \in L_{n+1} \mid \operatorname{Neg}\left(\pi^{-1}\right) \subseteq B\right\}
$$

into left cosets of $A_{n+1}$, and a set of distinguished coset representatives.

Lemma 4.5. Let $w \in S_{n+1}$. Then

$$
\ell_{L}(w)=\ell_{L}\left(s_{1} w\right)
$$

Proof.

$$
\operatorname{inv}\left(s_{1} w\right)= \begin{cases}\operatorname{inv}(w)+1 & \text { if } w^{-1}(1)<w^{-1}(2) \\ \operatorname{inv}(w)-1 & \text { if } w^{-1}(1)>w^{-1}(2)\end{cases}
$$

and

$$
\operatorname{del}_{B}\left(s_{1} w\right)= \begin{cases}\operatorname{del}_{B}(w)+1 & \text { if } w^{-1}(1)<w^{-1}(2) \\ \operatorname{del}_{B}(w)-1 & \text { if } w^{-1}(1)>w^{-1}(2)\end{cases}
$$

therefore

$$
\ell_{L}(w)=\operatorname{inv}(w)-\operatorname{del}_{B}(w)=\operatorname{inv}\left(s_{1} w\right)-\operatorname{del}_{B}\left(s_{1} w\right)=\ell_{L}\left(s_{1} w\right) .
$$

Lemma 4.6 (Main Lemma). Let $\pi \in L_{n+1}$. Then there exists a unique $\sigma \in L_{n+1}$ such that $u=\sigma^{-1} \pi \in A_{n+1}$ and $\operatorname{des}_{A}(\sigma)=0$. Moreover, $\operatorname{Des}_{A}(u)=\operatorname{Des}_{A}(\pi)$, $\operatorname{inv}(u)-$ $\operatorname{del}_{S}(u)=\operatorname{inv}(\pi)-\operatorname{del}_{B}(\pi)$, and $\operatorname{Neg}\left(\pi^{-1}\right)=\operatorname{Neg}\left(\sigma^{-1}\right)$.

Proof. Let $\sigma^{\prime} \in B_{n+1}$ be the increasing word with the letters of $\pi$. Clearly $\operatorname{inv}\left(\sigma^{\prime}\right)=$ $\operatorname{del}_{B}\left(\sigma^{\prime}\right)=0$ so by (2),$\ell_{L}\left(\sigma^{\prime}\right)=\sum_{i \in \operatorname{Neg}\left(\sigma^{\prime-1}\right)} i$.

For every $v \in S_{n+1}$ and $i, j \in[n+1]$,

$$
v(i)<v(j) \Longleftrightarrow\left(\sigma^{\prime} v\right)(i)<\left(\sigma^{\prime} v\right)(j)
$$

thus

$$
\operatorname{inv}\left(\sigma^{\prime} v\right)=\operatorname{inv}(v)
$$

and

$$
\operatorname{del}_{B}\left(\sigma^{\prime} v\right)=\operatorname{del}_{S}(v)
$$


By Remark 3.11, $\operatorname{Neg}\left(\left(\sigma^{\prime} v\right)^{-1}\right)=\operatorname{Neg}\left(v^{-1} \sigma^{\prime-1}\right)=\operatorname{Neg}\left(\sigma^{\prime-1}\right)$. Therefore for every $v \in$ $S_{n+1}$,

$$
\begin{aligned}
\ell_{L}\left(\sigma^{\prime} v\right) & =\operatorname{inv}\left(\sigma^{\prime} v\right)+\sum_{i \in \operatorname{Neg}\left(\left(\sigma^{\prime} v\right)^{-1}\right)} i-\operatorname{del}_{B}\left(\sigma^{\prime} v\right) \\
& =\sum_{i \in \operatorname{Neg}\left(\sigma^{\prime-1}\right)} i+\operatorname{inv}(v)-\operatorname{del}_{B}(v) \\
& =\ell_{L}\left(\sigma^{\prime}\right)+\ell_{L}(v)
\end{aligned}
$$

There are two possible cases to consider:

Case 1: $\sigma^{\prime} \in L_{n+1}$. Let $\sigma=\sigma^{\prime}$ and let $u=\sigma^{\prime-1} \pi$.

Using (6) we have for $1 \leq i \leq n-1$,

$$
\begin{aligned}
\ell_{L}\left(\sigma a_{i}\right) & =\ell_{L}\left(\sigma^{\prime} a_{i}\right) \\
& =\ell_{L}\left(\sigma^{\prime}\right)+\ell_{L}\left(a_{i}\right) \\
& >\ell_{L}\left(\sigma^{\prime}\right) \\
& =\ell_{L}(\sigma)
\end{aligned}
$$

and

$$
\begin{aligned}
\ell_{L}(\pi)-\ell_{L}\left(\pi a_{i}\right) & =\ell_{L}(\sigma u)-\ell_{L}\left(\sigma\left(u a_{i}\right)\right) \\
& =\ell_{L}\left(\sigma^{\prime} u\right)-\ell_{L}\left(\sigma^{\prime}\left(u a_{i}\right)\right) \\
& =\ell_{L}\left(\sigma^{\prime}\right)+\ell_{L}(u)-\ell_{L}\left(\sigma^{\prime}\right)-\ell_{L}\left(u a_{i}\right) \\
& =\ell_{L}(u)-\ell_{L}\left(u a_{i}\right) .
\end{aligned}
$$

Therefore $\operatorname{des}_{A}(\sigma)=0$ and $\operatorname{Des}_{A}(u)=\operatorname{Des}_{A}(\pi)$ as desired. From (41) and (5) we also get that

$$
\begin{aligned}
\operatorname{inv}(\pi)-\operatorname{del}_{B}(\pi) & =\operatorname{inv}(\sigma u)-\operatorname{del}_{B}(\sigma u) \\
& =\operatorname{inv}\left(\sigma^{\prime} u\right)-\operatorname{del}_{B}\left(\sigma^{\prime} u\right) \\
& =\operatorname{inv}(u)-\operatorname{del}_{S}(u) .
\end{aligned}
$$

Case 2: $\sigma^{\prime} s_{1} \in L_{n+1}$. Let $\sigma=\sigma^{\prime} s_{1}$ and let $u=s_{1} \sigma^{-1} \pi$.

Using (6) we have for $1 \leq i \leq n-1$,

$$
\begin{aligned}
\ell_{L}\left(\sigma a_{i}\right) & =\ell_{L}\left(\sigma^{\prime} s_{i+1}\right) \\
& =\ell_{L}\left(\sigma^{\prime}\right)+\ell_{L}\left(s_{i+1}\right) \\
& >\ell_{L}\left(\sigma^{\prime}\right) \\
& =\ell_{L}\left(\sigma^{\prime}\right)+\ell_{L}\left(s_{1}\right) \\
& =\ell_{L}\left(\sigma^{\prime} s_{1}\right) \\
& =\ell_{L}(\sigma)
\end{aligned}
$$


and, using also Lemma 4.5,

$$
\begin{aligned}
\ell_{L}(\pi)-\ell_{L}\left(\pi a_{i}\right) & =\ell_{L}\left(\sigma^{\prime} s_{1} u\right)-\ell_{L}\left(\sigma^{\prime}\left(s_{1} u a_{i}\right)\right) \\
& =\ell_{L}\left(\sigma^{\prime}\right)+\ell_{L}\left(s_{1} u\right)-\ell_{L}\left(\sigma^{\prime}\right)-\ell_{L}\left(s_{1} u a_{i}\right) \\
& =\ell_{L}\left(s_{1} u\right)-\ell_{L}\left(s_{1}\left(u a_{i}\right)\right) \\
& =\ell_{L}(u)-\ell_{L}\left(u a_{i}\right) .
\end{aligned}
$$

Therefore $\operatorname{des}_{A}(\sigma)=0$ and $\operatorname{Des}_{A}(u)=\operatorname{Des}_{A}(\pi)$ as desired. From (44) and (15) and Lemma 4.5 ,

$$
\begin{aligned}
\operatorname{inv}(\pi)-\operatorname{del}_{B}(\pi) & =\operatorname{inv}\left(\sigma^{\prime} s_{1} u\right)-\operatorname{del}_{B}\left(\sigma^{\prime} s_{1} u\right) \\
& =\operatorname{inv}\left(s_{1} u\right)-\operatorname{del}_{S}\left(s_{1} u\right) \\
& =\operatorname{inv}(u)-\operatorname{del}_{S}(u)
\end{aligned}
$$

In both cases, the fact that $\operatorname{Neg}\left(\pi^{-1}\right)=\operatorname{Neg}\left(\sigma^{-1}\right)$ follows by Remark 3.11 from the fact that $\pi^{-1}=u^{-1} \sigma^{-1}$ and $u \in A_{n+1}$.

To see that $\sigma$ is unique, suppose $\tilde{\sigma} \in L_{n+1}$ satisfies $\operatorname{des}_{A}(\tilde{\sigma})=0$ and $\tilde{u}=\tilde{\sigma}^{-1} \pi \in A_{n+1}$. Then $0=\operatorname{des}_{A}(\tilde{\sigma})=\operatorname{des}_{A}\left(\sigma u \tilde{u}^{-1}\right)\left(\right.$ since $\left.\tilde{\sigma}=\sigma u \tilde{u}^{-1}\right)$, so for $1 \leq i \leq n-1$,

$$
\begin{aligned}
0 & \leq \ell_{L}\left(\sigma u \tilde{u}^{-1} a_{i}\right)-\ell_{L}\left(\sigma u \tilde{u}^{-1}\right) \\
& =\ell_{L}(\sigma)+\ell_{L}\left(u \tilde{u}^{-1} a_{i}\right)-\ell_{L}(\sigma)-\ell_{L}\left(u \tilde{u}^{-1}\right) \\
& =\ell_{L}\left(u \tilde{u}^{-1} a_{i}\right)-\ell_{L}\left(u \tilde{u}^{-1}\right) \\
& =\ell_{A}\left(u \tilde{u}^{-1} a_{i}\right)-\ell_{A}\left(u \tilde{u}^{-1}\right),
\end{aligned}
$$

whence $u \tilde{u}^{-1}=1$, i.e. $\sigma=\tilde{\sigma}$.

Let $T=\left\{\sigma \in L_{n+1} \mid \operatorname{des}_{A}(\sigma)=0\right\}$.

Corollary 4.7. 1. For every $B \subseteq[n+1]$ there exists a unique $\sigma \in T$ such that $B=$ $\operatorname{Neg}\left(\sigma^{-1}\right)$.

2. For every $B \subseteq[n+1]$,

$$
\left\{\pi \in L_{n} \mid \operatorname{Neg}\left(\pi^{-1}\right) \subseteq B\right\}=\biguplus_{u \in A_{n+1}}\left\{\sigma u \mid \sigma \in T, \operatorname{Neg}\left(\sigma^{-1}\right) \subseteq B\right\},
$$

where $\uplus$ denotes disjoint union.

Corollary 4.8. Let $\pi \in L_{n+1}$, and write $\pi=\sigma u$ with $\sigma$ and $u$ like in Lemma 4.6. Then $\ell_{L}(\pi)=\ell_{A}(u)+\sum_{i \in \operatorname{Neg}\left(\pi^{-1}\right)} i$.

Proof. By (2), Lemma 4.6 and Proposition 3.14,

$$
\begin{aligned}
\ell_{L}(\pi) & =\operatorname{inv}(\pi)-\operatorname{del}_{B}(\pi)+\sum_{i \in \operatorname{Neg}\left(\pi^{-1}\right)} i \\
& =\operatorname{inv}(u)-\operatorname{del}_{S}(u)+\sum_{i \in \operatorname{Neg}\left(\pi^{-1}\right)} i \\
& =\ell_{A}(u)+\sum_{i \in \operatorname{Neg}\left(\pi^{-1}\right)} i .
\end{aligned}
$$


Proof of Proposition 4.1. From Corollary 4.7 Lemma 4.6 and Theorem 4.4 .

$$
\begin{aligned}
\sum_{\substack{\pi \in L_{n+1} \\
\operatorname{Neg}\left(\pi^{-1}\right) \subseteq B}} q^{\mathrm{nrmaj}_{L_{n+1}}(\pi)} & =\sum_{\substack{\sigma \in T \\
\operatorname{Neg}\left(\sigma^{-1}\right) \subseteq B}} \sum_{u \in A_{n+1}} q^{\mathrm{nrmaj}_{L_{n+1}}(\sigma u)} \\
& =\sum_{\substack{\sigma \in T \\
\operatorname{Neg}\left(\sigma^{-1}\right) \subseteq B}} \sum_{u \in A_{n+1}} q^{\mathrm{rmaj}_{L}(\sigma u)+\sum_{i \in \operatorname{Neg}\left((\sigma u)^{-1}\right)^{i}}} \\
& =\sum_{\substack{\sigma \in T \\
\operatorname{Neg}\left(\sigma^{-1}\right) \subseteq B}} q^{\sum_{i \in \operatorname{Neg}\left(\sigma^{-1}\right)} i} \sum_{u \in A_{n+1}} q^{\mathrm{rmaj}_{A}(u)} \\
& =\sum_{C \subseteq B} q^{\sum_{i \in C} i} \sum_{u \in A_{n+1}} q^{\mathrm{rmaj}_{A}(u)} \\
& =\prod_{i \in B}\left(1+q^{i}\right) \prod_{i=1}^{n-1}\left(1+q+\cdots+q^{i-1}+2 q^{i}\right) .
\end{aligned}
$$

By similar considerations, this time invoking the other equality in Theorem 4.4 ,

$$
\begin{aligned}
\sum_{\substack{\pi \in L_{n+1} \\
\operatorname{Neg}\left(\pi^{-1}\right) \subseteq B}} q^{\ell_{L}(\pi)} & =\sum_{\substack{\sigma \in T \\
\operatorname{Neg}\left(\sigma^{-1}\right) \subseteq B}} \sum_{u \in A_{n+1}} q^{\ell_{L}(\sigma u)} \\
& =\sum_{\substack{\sigma \in T \\
\operatorname{Neg}\left(\sigma^{-1}\right) \subseteq B}} \sum_{u \in A_{n+1}} q^{\operatorname{inv}(\sigma u)+\sum_{i \in \operatorname{Neg}((\sigma u)-1)} i-\operatorname{del}_{B}(\sigma u)} \\
& =\sum_{\substack{\sigma \in T \\
\operatorname{Neg}\left(\sigma^{-1}\right) \subseteq B}} q^{\sum_{i \in \operatorname{Neg}\left(\sigma^{-1}\right)} i} \sum_{u \in A_{n+1}} q^{\operatorname{inv}(u)-\operatorname{del}_{S}(u)} \\
& =\sum_{C \subseteq B} q^{\sum_{i \in C} i} \sum_{u \in A_{n+1}} q^{\ell_{A}(u)} \\
& =\prod_{i \in B}\left(1+q^{i}\right) \prod_{i=1}^{n-1}\left(1+q+\cdots+q^{i-1}+2 q^{i}\right) .
\end{aligned}
$$

\section{Even-signed Even Permutations}

We denote by $D_{n}$ the group of even-signed permutations, that is the subgroup of $B_{n}$ consisting of all the signed permutations having an even number of negative entries in their window notation. Equivalently,

$$
D_{n}=\left\{\sigma \in B_{n} \mid \# \operatorname{Neg}\left(\sigma^{-1}\right) \text { is even }\right\} .
$$

$D_{n}$ is a Coxeter group of type $D$, generated by $\tilde{s}_{0}, s_{1}, \ldots, s_{n-1}$, where $\tilde{s}_{0}=s_{0} s_{1} s_{0}=$ $[-2,-1,3, \ldots, n]$ (see, for example, [4, $\S 8.2]$ ). 
Following Biagioli [3], we define the D-length of $\sigma \in D_{n}$ by

$$
\ell_{D}(\sigma)=\ell_{B}(\sigma)-\# \operatorname{Neg}(\sigma),
$$

which is also the length of a reduced expression for $\sigma$ in the above generators (see [4, $\S 8.2$ ] for a proof), and we let

$$
\operatorname{dmaj}(\sigma)=\operatorname{maj}_{B}(\sigma)-\# \operatorname{Neg}(\sigma)+\sum_{i \in \operatorname{Neg}\left(\sigma^{-1}\right)} i .
$$

Biagioli proved the following $D_{n}$-analogue of MacMahon's theorem.

Proposition 5.1 (see [3, Proposition 3.1]).

$$
\sum_{\sigma \in D_{n}} q^{\operatorname{dmaj}(\sigma)}=\sum_{\sigma \in D_{n}} q^{\ell_{D}(\sigma)}
$$

Let

$$
\operatorname{drmaj}_{n}(\sigma)=\operatorname{rmaj}_{B_{n}}(\sigma)-\# \operatorname{Neg}(\sigma)+\sum_{i \in \operatorname{Neg}\left(\sigma^{-1}\right)} i .
$$

Since the involution $\phi$ from Lemma 3.19 satisfies the condition (3), dmaj and drmaj ${ }_{n}$ are equidistributed on $D_{n}$, hence we can replace dmaj with drmaj ${ }_{n}$ in Proposition 5.1 .

Let $(L \cap D)_{n+1}=L_{n+1} \cap D_{n+1}$, the group of even-signed even permutations on $\pm 1, \ldots, \pm(n+1)$, and let

$$
\ell_{(L \cap D)}(\pi)=\ell_{D}(\pi)-\operatorname{del}_{B}(\pi)
$$

and

$$
\operatorname{drmaj}_{(L \cap D)_{n+1}}(\pi)=\operatorname{rmaj}_{L_{n+1}}(\pi)-\# \operatorname{Neg}(\pi)+\sum_{i \in \operatorname{Neg}\left(\pi^{-1}\right)} i .
$$

\section{Proposition 5.2.}

$$
\sum_{\pi \in(L \cap D)_{n+1}} q^{\operatorname{drmaj}_{(L \cap D)_{n+1}}(\pi)}=\sum_{\pi \in(L \cap D)_{n+1}} q^{\ell_{(L \cap D)}(\pi)}
$$

Proof. From the definitions and from Corollary 4.3 we have for every $i$

$$
\begin{aligned}
\sum_{\substack{\pi \in L_{n+1} \\
\# \operatorname{Neg}\left(\pi^{-1}\right)=2 i}} q^{\operatorname{drmaj}_{(L \cap D)_{n+1}}(\pi)}=\sum_{\substack{\pi \in L_{n+1} \\
\# \operatorname{Neg}\left(\pi^{-1}\right)=2 i}} q^{\operatorname{nrmaj}_{L_{n+1}}(\pi)-\# \operatorname{Neg}(\pi)} \\
=q^{-2 i} \sum_{\substack{B \subseteq[n+1] \\
|B|=2 i}} \sum_{\substack{\pi \in L_{n+1} \\
\operatorname{Neg}\left(\pi^{-1}\right)=B}} q^{\operatorname{nrmaj}_{L_{n+1}}(\pi)} \\
=q^{-2 i} \sum_{\substack{B \subseteq[n+1] \\
|B|=2 i}} \sum_{\substack{\pi \in L_{n+1} \\
\operatorname{Neg}\left(\pi^{-1}\right)=B}} q^{\ell_{L}(\pi)} \\
=\sum_{\substack{\pi \in L_{n+1} \\
\# \operatorname{Neg}\left(\pi^{-1}\right)=2 i}} q^{\ell_{L}(\pi)-\# \operatorname{Neg}(\pi)} \\
=\sum_{\substack{\pi \in L_{n+1} \\
\# \operatorname{Neg}\left(\pi^{-1}\right)=2 i}} q^{\ell_{(L \cap D)}(\pi)} .
\end{aligned}
$$


Taking the sum over all $i$ we get the desired equality.

\section{Open Problems}

The following questions arise quite naturally when considering what is known for $S_{n}$ and $B_{n}$ and comparing our results for $L_{n+1}$ with the results for $A_{n+1}$ from [8]. However, they remain open.

1. Is it possible to define a descent number $\operatorname{des}_{L}$ on $L_{n+1}$ for which a theorem like Corollary 1.11 in [8], that is

$$
\sum_{\pi \in L_{n+1}} q_{1}^{\operatorname{nrmaj}_{L_{n+1}}(\pi)} q_{2}^{\operatorname{des}_{L}\left(\pi^{-1}\right)}=\sum_{\pi \in L_{n+1}} q_{1}^{\ell_{L}(\pi)} q_{2}^{\operatorname{des}_{L}\left(\pi^{-1}\right)}
$$

holds?

2. The statistic $\operatorname{del}_{S}$ (resp. $\operatorname{del}_{A}$ ), as defined in [8], has an algebraic interpretation as the number of occurrences of $s_{1}$ (resp. $a_{1}^{ \pm 1}$ ) in the canonical presentation of an element. Is there an interpretation of $\operatorname{del}_{B}(\sigma)$ based on counting occurrences of generators in the $B$ canonical presentation of $\sigma$ ? Alternatively, is there another canonical presentation of $B_{n}$ for which $\operatorname{del}_{B}$ has such a meaning?

3. For $\pi \in L_{n+1}$ one can define length $(\pi)$, the length of $\pi$ with respect to the set of generators $\left\{a_{0}, a_{1}, \ldots, a_{n-1}\right\}$, and then proceed to define a notion of descent. Is there a closed formula for length $(\pi)$ ? How does it relate to $\ell_{L}(\pi)$ ?

\section{Acknowledgements}

I would like to thank my thesis advisor, Amitai Regev, for suggesting the topic and for his helpful remarks on preliminary versions of this paper. I am also thankful to the anonymous referee for making useful comments on the organization of the paper, referring me to known results in the literature, and suggesting the inclusion of open problems.

\section{References}

[1] R. M. Adin, F. Brenti and Y. Roichman, Descent numbers and major indices for the hyperoctahedral group, Adv. in Appl. Math. 27 (2001), 210-224.

[2] E. Bagno, Combinatorial parameters on classical groups, Ph. D. Thesis, Bar-Ilan University, 2004.

[3] R. Biagioli, Major and descent statistics for the even-signed permutation group, Adv. in Appl. Math. 31 (2003), 163-179. 
[4] A. Björner and F. Brenti, Combinatorics of Coxeter Groups, Graduate Texts in Mathematics, Springer-Verlag, to appear.

[5] D. M. Goldschmidt, Group characters, symmetric functions, and the Hecke algebra, Amer. Math. Soc. University Lecture Series, Vol. 4 (1993).

[6] P. A. MacMahon, Combinatory Analysis I-II, Cambridge Univ. Press, London and New York, 1916. (Reprinted by Chelsea, New York, 1960).

[7] H. Mitsuhashi, The q-analogue of the alternating group and its representations, J. Alg. 240 (2001), 535-558.

[8] A. Regev and Y. Roichman, Permutation statistics on the alternating group, Adv. in Appl. Math. 33 (2004), 676-709. doi:10.1016/j.aam.2003.10.004

[9] A. Regev and Y. Roichman, Statistics on wreath products and generalized binomialStirling numbers, preprint, 2004, arXiv:math.C0/0404354. 